\title{
The Protective Role of Celastrol in Renal Ischemia-Reperfusion Injury by Activating Nrf2/HO-1, PI3K/AKT Signaling Pathways, Modulating NF-kb Signaling Pathways, and Inhibiting ERK Phosphorylation
}

\author{
Nancy S. Younis $\mathbb{D}^{1} \cdot$ Amal M. H. Ghanim ${ }^{2}$ \\ Received: 1 October 2021 / Accepted: 26 January 2022 / Published online: 14 February 2022 \\ (c) The Author(s) 2022
}

\begin{abstract}
Celastrol, a natural triterpenoid derived from Tripterygium wilfordii, possesses numerous biological effects. We investigated celastrol's antioxidant potential through nuclear factor erythroid 2-related factor 2 (Nrf2)/heme oxygenase 1 (HO-1) and its effect on phosphoinositide 3-kinase (PI3K)/protein kinase B (AKT) signaling, nuclear factor-kappa B (NF-kB) pathways, and extracellular signal-regulated kinase (ERK) activation in kidney ischemia-reperfusion injury (IRI) rat model. Rats were given celastrol $2 \mathrm{mg} / \mathrm{kg}$ orally for 1 week before subjection to renal ischemia-reperfusion surgery. Kidney functions, renal

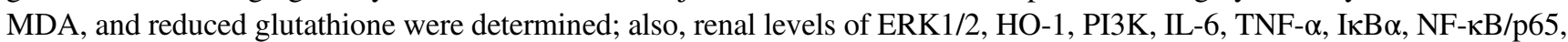
and cleaved caspase-3 were measured. In addition, gene expression of kidney injury molecule-1 (KIM-1), Nrf-2, and AKT were determined. Celastrol pretreatment attenuated oxidative stress and increased Nrf2 gene expression and HO-1 level. Also, it activated the PI3K/AKT signaling pathway and decreased the p-ERK:t- ERK ratio and NFKBp65 level, with a remarkable decrease in inflammatory cytokines and cleaved caspase-3 levels compared with those in renal IRI rats. Conclusively, celastrol showed a reno-protective potential against renal IRI by suppressing oxidative stress through enhancing the Nrf2/HO-1 pathway, augmenting cell survival PI3K/AKT signaling pathways, and reducing inflammation by inhibiting NF- $\mathrm{KB}$ activation.
\end{abstract}

Keywords Celastrol $\cdot$ Renal Ischemia-Reperfusion $\cdot \mathrm{Nrf2/HO}-1 \cdot \mathrm{PI} 3 \mathrm{~K} / \mathrm{AKT} \cdot$ ERK phosphorylation

$\begin{array}{ll}\text { Abbreviation } \\ \text { AKT } & \text { Protein kinase B } \\ \text { ERK } & \text { Extracellular signal-regulated kinase } \\ \text { HO-1 } & \text { Heme oxygenase 1 } \\ \text { IL-6 } & \text { Interleukin-6 } \\ \text { IRI } & \text { Ischemia-reperfusion injury } \\ \text { KIM-1 } & \text { Kidney injury molecule } \\ \text { NF-kB } & \text { Nuclear factor-kappa B } \\ \text { Nrf2 } & \text { Nuclear factor erythroid 2-related factor 2 } \\ \text { PI3K } & \text { Phosphoinositide 3-kinase } \\ \text { TNF- } \alpha & \text { Tumor necrosis alpha }\end{array}$

Nancy S. Younis

nyounis@kfu.edu.sa

1 Department of Pharmaceutical Sciences, College of Clinical Pharmacy, King Faisal University, Al-Ahsa, Kingdom of Saudi Arabia

2 Department of Biochemistry, Faculty of Pharmacy, Fayoum University, Fayoum, Egypt

\section{Introduction}

Pharmacological bioactive molecules obtained from medicinal plants have attracted substantial importance lately due to their robust, unique, diverse actions. Celastrol is a pentacyclic triterpene extracted from Tripterygium wilfordi (TW) [1], with the chemical structure provided in Fig. 1. Celastrol attains numerous pharmacological actions, including antioxidant and anti-inflammatory $[2,3]$. It has been proven that celastrol suppresses several proinflammatory cytokines, including tumor necrosis alpha (TNF- $\alpha$ ), interleukins (IL-2, IL-8), interferon-gamma (IFN$\gamma$ ), proinflammatory enzymes, nitric oxide synthase, nuclear factor-kappa B (NFkB), and adhesion molecules [4, 5]. Thus, it showed promising actions in diverse inflammatory diseases such as rheumatoid arthritis [6], Crohn's disease [7], ulcerative colitis [8], and asthma [9]. Additionally, celastrol was proven to possess anti-obesity [10], neuroprotective effect [11] and anti-diabetic [12]. Moreover, Celastrol's anticancer potential was confirmed in many studies by promoting apoptosis and inhibiting angiogenesis, 


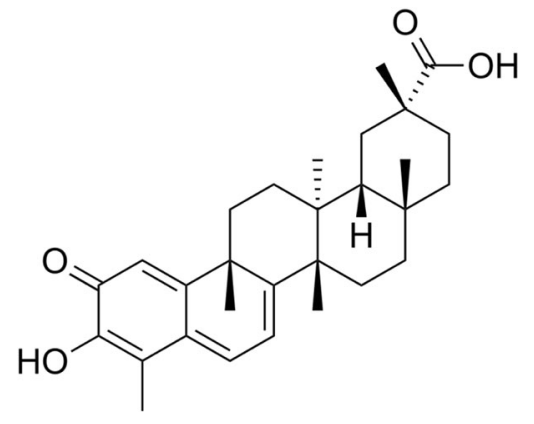

Fig. 1 Chemical structure of celastrol

proliferation, and cell invasion [13-15]. Due to such diverse pharmacological actions, numerous clinical trials were conducted using TW plant extract. Furthermore, randomized clinical trials on patients with various diseases, such as rheumatoid arthritis [16], solid tumor [17], diabetic nephropathy [18], and renal transplantation [19] in attempts to evaluate celastrol therapeutically for a more in-depth investigation on Castrol's actions on the kidney. A study by Zhang, Chen [20] showed that celastrol reduces blood contents of creatinine and urea nitrogen in a diabetic nephropathy rat model and reduces urinary protein excretion, improving pathological renal injury by regulating mitogen-activated protein kinase (MAPK)/NF-кB pathway. Another research group proved that celastrol treatment could attenuate unilateral ureteral obstruction induced mouse renal fibrosis and folic acid-induced mouse renal fibrosis [21].

In cisplatin-induced kidney injury, Celastrol treatment improved renal function, kidney morphology, oxidative stress, and suppressed renal tubular injury markers; kidney injury molecule (KIM-1) and neutrophil gelatinaseassociated lipocalin NGAL). Moreover, it inhibited renal apoptosis and inflammation by deterring tubular cell apoptosis and NF- $\mathrm{KB}$ activation and enhancing mitochondrial function [22]. Furthermore, Celastrol improved ischemiareperfusion induced injury (IRI) as it significantly suppressed renal function markers, oxidative stress and prevented the expression of proinflammatory mediators, which was associated with the suppression of nuclear translocation of NF-KB subunit p65 thus, alleviating renal tubular damage [23]. In this study, we aimed at investigating the antioxidant potential of celastrol through its effect on the nuclear factor erythroid 2-related factor 2 (Nrf2)/heme oxygenase 1 (HO-1) pathway. This pathway is considered a key regulator of cellular antioxidant defense mechanism that may be a good target for new therapeutic approaches to alleviate kidney cell damage induced by IR and the accompanying oxidative stress. Likewise, our study attempted underlining celastrol effect on cell survival mediating pathway; phosphoinositide 3-kinase (PI3K)/protein kinase B (AKT). In addition, exploring Celastrol's anti-inflammatory and antiapoptotic effects through its effect on extracellular signal-regulated kinase (ERK) as a MAPK family member and NF- $\mathrm{KB}$ transcription factor is greatly associated with inflammation and apoptosis during renal injury.

\section{Material and Methods}

\section{Animals}

Forty-eight-week-old adult male Swiss albino rats weighing $200 \pm 20 \mathrm{~g}$ were obtained from the Faculty of Pharmacy, Delta University, Egypt. The rats were accommodated in a controlled environment $\left(24 \pm 1{ }^{\circ} \mathrm{C}\right.$, $50 \pm 10$ humidity) and a $12 \mathrm{~h}: 12 \mathrm{~h}$ light:dark cycle with free access to food and water ad lib. Rat care and experimental techniques were permitted and approved by the Institutional Animal Care and Use Committee (IACUC) at the Faculty of Pharmacy, Delta University for Science and Technology. Ethical approval number: FPDU 6/2019. All experiments were conducted following relevant guidelines and regulations.

\section{Experimental Design and Surgery}

Rats were randomized into four groups ( $n=10$ each). First, sham-operated group, in which rats were subjected to bilateral renal artery separation with no clamping (Sham). Second, sham-celastrol-operated group, in which rats were given celastrol $2 \mathrm{mg} / \mathrm{kg}$, orally [22] for 1 week, then subjected to bilateral renal artery separation with no clamping (Sham + cela). Third, renal IR group, in which kidney injury was induced in rats by renal ischemia-reperfusion surgery (IRI). Finally, renal IR + celastrol group, in which animals were administered celastrol $2 \mathrm{mg} / \mathrm{kg}$ orally for 1 week before renal IR surgery (IRI + cela). Animals were anesthetized with $2 \%$ pentobarbital sodium $(50 \mathrm{mg} / \mathrm{kg}$, i.p.) and maintained on a heating pad to sustain rats' body temperature at $37{ }^{\circ} \mathrm{C}$ to perform renal IR surgery. Then, nephrectomy of the left kidney was performed, and clamping the right bilateral renal artery using a nontraumatic artery clamp for $30 \mathrm{~min}$ followed by $24 \mathrm{~h}$ reperfusion to induce renal IRI [24].

Twenty-four hours after renal reperfusion, animals were sacrificed by decapitation under anesthesia, and blood samples were collected from different experimental groups. Serum samples were obtained via $15 \mathrm{~min}$ of blood centrifugation at $5000 \mathrm{rpm}$ and were stored at $-20^{\circ} \mathrm{C}$ for subsequent measurements. The right kidney was rapidly removed and separated into two parts; the first part was homogenized and kept at $-80{ }^{\circ} \mathrm{C}$ for biochemical analyses. The second part was kept in $10 \%$ formalin for histopathological examination. 


\section{Assessing Kidney Function and Oxidative Stress}

Kidney function was assessed by measuring serum creatinine (Cat. No; 235 001) and blood urea nitrogen (BUN) (Cat. No; 310 100) levels using a commercially available kit obtained from Spectrum Diagnostics, Egypt. For oxidative stress assessment, renal lipid peroxidation assay was performed by measurement of MDA level in kidney tissue homogenate by commercially available kit (Cat. no; LIP39K01) purchased from Eagle Biosciences, Inc. (Boston, MA, USA). Also, renal reduced glutathione (GSH) assay was performed using the kit attained from BioVision incorporated (Milpitas, CA, USA) (Cat. No; K464-100)

\section{Enzyme-Linked Immunosorbent Assay Determination of the Renal Level of ERK1/2, HO-1, PI3K, IL-6, TNF-a, IKBa, NF-KB/p65, and Cleaved Caspase-3}

The following parameters' concentration was determined in renal tissue using demonstrated enzyme-linked immunosorbent assay (ELISA) kits following the manufacturers' protocols. Phosphorylated ERK1/2 and Total ERK1/2 ELISA kits were obtained (Abcam, Cambridge, United Kingdom) (Cat. no; ab176660). Then, antibody mix was added to samples, and TMB substrate was added and catalyzed by horseradish peroxidase (HRP), generating blue coloration. This reaction was stopped by adding a stop solution, and the color intensity was measured at $450 \mathrm{~nm}$. I $\kappa \mathrm{B} \alpha$ ELISA Kits were obtained from ELISA Genie (Dublin, Ireland) (Cat. No; RTFI00897); a biotin-detection antibody and TMB substrate were added after the stop solution. Optical density absorbance was read at $450 \mathrm{~nm}$. Heme oxygenase-1 (HO-1) ELISA Kit was bought from BioVision incorporated (Milpitas, CA, USA) (Cat. no; E4525-100); biotin-detection antibodies were added to samples, and then 90- $\mu \mathrm{l}$ TMB was added and incubated ( $37{ }^{\circ} \mathrm{C}$ in the dark for $15-30 \mathrm{~min}$ ). Shades of blue were seen and the stop solution was added; the result was read at $450 \mathrm{~nm}$. Interleukin 6 ELISA Kit was acquired from Life Span BioSciences Inc (Seattle, WA, USA) (Cat. no; LSF25921) for the assay based on double antibody sandwich method with chemiluminescence detection. The supplied plate has been pre-coated with a target-specific capture antibody. Samples and a biotin-conjugated detection antibody were added. An Avidin- HRP conjugate was then bound to the biotin, and unbound Avidin-HRP conjugate was washed away. A chemiluminescent substrate was then added, and the relative light units of each well were measured. PI3K ELISA Kits were obtained from MyBioSource (San Diego, California, United States) (Cat. no; MBS 702819); PI3K ELISA kit used double-sandwich ELISA technique. In which samples and biotin labeling antibody were added followed by Avidin-peroxidase conjugate addition. TMB substrate was used for color development, and the optical density was detected at $450 \mathrm{~nm}$. TNF- $\alpha$ ELISA Kits were obtained from MyBioSource (San Diego, California, United States) (Cat. no; MBS355371); Biotinantibody was added to samples, and HRP-avidin was added to each well, then TMB Substrate was added after washing, and finally $50 \mu$ of stop solution was added. The optical density was determined at $450 \mathrm{~nm}$. Cleaved caspase-3 ELISA Kits were obtained from MyBioSource (San Diego, California, United States) (Cat. no; MBS7244630) for the polyclonal anti-A-caspase-3 antibody and an A-caspase-3HRP conjugate. The wells were then incubated with a HRP enzyme substrate to form a blue complex. The intensity of the color is measured at $450 \mathrm{~nm}$.

NF- $\kappa B$ p65 ELISA Kit was obtained from Novus Biologicals, LLC (Littleton, Colorado, United States) (Cat. no; NBP2-29661); captured antibodies were added to each well and incubated overnight at $4{ }^{\circ} \mathrm{C}$. Blocking buffer was added and incubated for $30 \mathrm{~min}$. Positive and negative controls and samples were used, and the detecting antibody was added after washing. This is followed by diluted secondary antibody addition. After washing, pNPP substrate was added, and the color developed was detected at $405 \mathrm{~nm}$.

\section{Histological Staining}

Kidney tissue of different groups were fixed with $10 \%$ formalin saline at room temperature and embedded in paraffin. 4- $\mu \mathrm{m}$ thick sections of kidney tissue were cut and stained with hematoxylin and eosin (H \& E) to determine morphological changes in the kidney. Additionally, kidney sections were subjected to PAS staining and scored under a microscope. Finally, renal tissue images were captured under a light microscope.

\section{Quantitative Real-time PCR for KIM-1, Nrf-2, and AKT Determination}

Total RNA was isolated from the homogenized kidney tissue with a total RNA purification kit provided by Jena Bioscience (Munich, Germany) and stored at $-80^{\circ} \mathrm{C}$. RNA was converted into complementary DNA by a cDNA archive kit (Applied Biosystems, Foster City, California, USA). qPCR was performed using GoTaq PCR master mix (Promega Co., Madison, USA). A protocol that included an initial denaturation step at $95^{\circ} \mathrm{C}$ for $10 \mathrm{~min}$, followed by 40 cycles of denaturing at $95^{\circ} \mathrm{C}$ for $15 \mathrm{~s}$, annealing and extension at $60^{\circ} \mathrm{C}$ for $1 \mathrm{~min}$ then $60^{\circ} \mathrm{C}$ for $30 \mathrm{~s}$ were performed on a StepOne Real-Time PCR System (Applied Biosystems, Foster City, California, USA). Descriptions of the sequences of oligonucleotide primers used is shown in Table 1 
Table 1 Primer sequences used in this study were as follows

\begin{tabular}{|c|c|}
\hline Markers (gene bank accession number) & Primer sequence $\left(5^{\prime}\right.$ to $\left.3^{\prime}\right)$ \\
\hline KIM-1 (AF035963.1) & $\begin{array}{l}\text { 5'-TTTGGATCTGTACCCAGTGCTT-3'(sense), } \\
\text { 5'-CAAGGCCAGCCCTCTAATGG-3' (antisense }\end{array}$ \\
\hline Nrf-2 (NM_031789.2) & $\begin{array}{l}\text { 5'-TTGTAGATGACCATGAGTCGC-3'(sense), } \\
\text { 5'-TGTCCTGCTGTATGCTGCTT-3' (antisense) }\end{array}$ \\
\hline AKT (NM_033230.2) & $\begin{array}{l}\text { 5'-GCCCAACACCTTCATCATCC-3'(sense), } \\
\text { 5'-:GTCTCCTCCTCCTGCCGTTT-3' (antisense) }\end{array}$ \\
\hline$\beta$-actin (NM001106409.1) as an internal control & 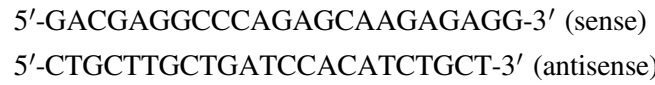 \\
\hline
\end{tabular}

KIM kidney injury molecule -1, Nrf2 nuclear factor erythroid 2-related factor 2, AKT protein kinase B

\section{Statistical Analysis}

All data are presented as the mean \pm standard deviation (SD). Between-group differences were detected via oneway analysis of variance. Finally, these data were analyzed using GraphPad Prism v.7.0 (GraphPad Software, Inc., San Diego, CA). $P<0.05$ indicated statistical significance.

\section{Results}

\section{Celastrol Enhanced Kidney Function and Renal Oxidative Stress}

As shown in Table 2, IRI caused kidney function deterioration indicated by a significant increase in serum creatinine and BUN level compared with the sham group $(P<0.001)$. Also, renal IRI induced a significant increase in malondialdehyde (MDA) level and a remarkable decrease in reduced glutathione (GSH) level compared with shamoperated rats $(P<0.001)$. However, celastrol pretreatment significantly decreased serum creatinine and BUN levels, reduced the elevated MDA level, restored the depleted GSH level, and compared with the renal IRI group $(P<0.001)$.

\section{Celastrol Enhanced Nrf2 Gene Expression and HO-1 Level and Suppressed KIM-1, Gene Expression}

Renal ischemia-reperfusion caused severe kidney damage confirmed by the significant upregulation of KIM-1 gene expression compared with sham-operated rats $(P<0.001)$. This is accompanied by enhancement of Nrf2 gene expression and rise in renal HO-1 level as a self-protective response to the injury. However, celastrol pretreatment displayed a notable renal protective and antioxidant effect by significant suppression of KIM-1 gene expression and remarkable upregulation of Nrf2 gene expression and subsequent elevation of HO-1 levels compared with the renal IRI group $(P<0.001)$ (Fig. 2a-c).

\section{Celastrol Activated PI3K/AKT Signaling}

Ischemic injury triggered a significant suppression in cell survival mediating pathway; PI3K/AKT via a remarkable decrease in PI3K level and AKT gene expression $(P<$ 0.001). Notably, celastrol treatment activates the PI3K/AKT signaling pathway through PI3K-level preservation and AKT gene expression enhancement compared with the IRI group $(P<0.001)$ (Fig. 3a, b).

\section{Celastrol Enhanced IкBa Level and Suppressed p- ERK1/2, t-ERK1/2, NFKBp65 Levels}

Kidney injury induced by ischemia-reperfusion significantly enhanced ERK 1/2 phosphorylation and increased p-ERK: t- ERK ratio compared with the sham group $(P<0.001)$. Moreover, renal IRI rats exhibited a significant increase in $\mathrm{NF \kappa Bp} 65$ with significant suppression of $\mathrm{Nf \kappa B}$ inhibitor protein; IкB $\alpha$ levels $(P<0.001)$. These findings were reversed upon celastrol treatment; p-ERK: t- ERK ratio and NFKBp65 level were significantly decreased $(P<0.05$ and $P<0.001$, respectively) with a remarkable increase in IкB $\alpha$ level compared with the renal IRI group $(P<0.001)$ (Fig. 4a-c).

\section{Celastrol Suppressed IL-6, TNFa, and Cleaved Caspase-3 Levels}

As presented in (Fig. 5a-c), kidney injury was accompanied by a significant increase in inflammatory cytokines, IL-6 and TNF $\alpha$ levels $(P<0.001)$. Also, ischemiareperfusion significantly increases the apoptotic marker cleaved caspase-3 level compared with sham-operated rats $(P<0.001)$. Celastrol treatment exhibited a pronounced anti-inflammatory and antiapoptotic effect shown by the remarkable decrease in IL- 6 , TNF $\alpha$, and cleaved caspase- 3 levels compared with the renal IRI group $(P<0.001)$. 
Table 2 Effect of celastrol treatment $(2 \mathrm{mg} / \mathrm{kg}$; P.O) on serum creatinine, BUN, renal MDA and reduced glutathione levels in renal IRI rats

\begin{tabular}{llrll}
\hline & Sham & Sham + cela & IRI & IRI + cela \\
\hline Creatinine $(\mathrm{mg} / \mathrm{dl})$ & $0.838 \pm 0.19$ & $0.88 \pm 0.14$ & $3.436 \pm 0.066^{+++}$ & $1.584 \pm 0.17^{+++* * *}$ \\
BUN $(\mathrm{mg} / \mathrm{dl})$ & $10.52 \pm 3.11$ & $17.22 \pm 3.87$ & $67.81 \pm 12.16^{+++}$ & $34.98 \pm 8.03^{++* * *}$ \\
MDA $(\mu \mathrm{M} / \mathrm{mg}$ tissue protein) & $0.565 \pm 0.12$ & $0.692 \pm 0.09$ & $3.245 \pm 0.41^{+++}$ & $1.158 \pm 0.19^{++* * *}$ \\
$\begin{array}{l}\text { Reduced GSH }(\mu \mathrm{M} / \mathrm{mg} \text { tissue } \\
\text { protein) }\end{array}$ & $404.2 \pm 50.2$ & $364.5 \pm 57.6$ & $150 \pm 28.1^{+++}$ & $302.7 \pm 50.2^{++* * *}$ \\
\hline
\end{tabular}

All results were expressed as mean $\pm \mathrm{SD} ; n=6$ each

$M D A$ malondialdehyde, GSH glutathione, $B U N$ blood urea nitrogen, IRI ischemia reperfusion injury ${ }^{+++} P<0.001,{ }^{++} P<0.01$ compared with sham group and ${ }^{* * *} P<0.001$ compared with renal IRI group
Fig. 2 Effect of celastrol treatment $(2 \mathrm{mg} / \mathrm{kg}$; PO) on gene expression of a KIM-1, b Nrf2, and $\mathbf{c}$ HO-1 levels in renal IRI rats. All results were expressed as mean $\pm \mathrm{SD} ; n=6$ in each. KIM-1 kidney injury molecule1, Nrf2 nuclear factor erythroid 2-related factor 2, HO-1 hemoxygenase-1, IRI ischemiareperfusion injury. ${ }^{+++} P<$ $0.001,{ }^{+} P<0.05$ compared with the sham group, ${ }^{* * * *} P<0.001$ compared with the renal IRI group

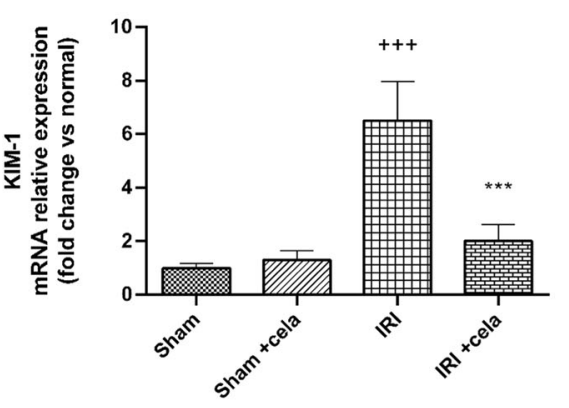

b)

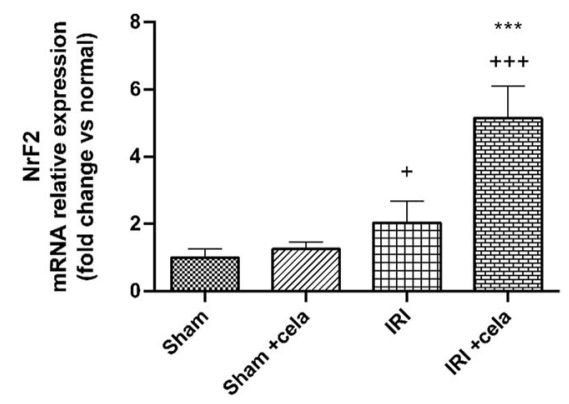

c)

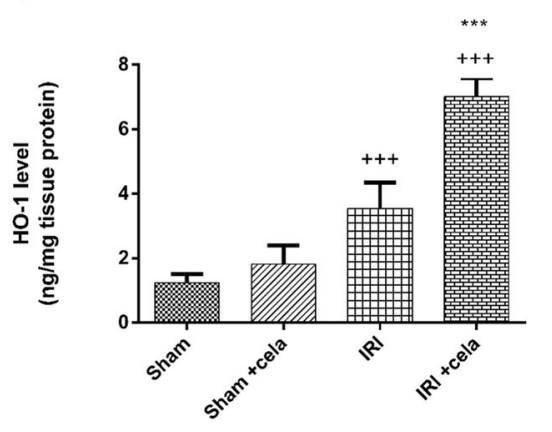

a)

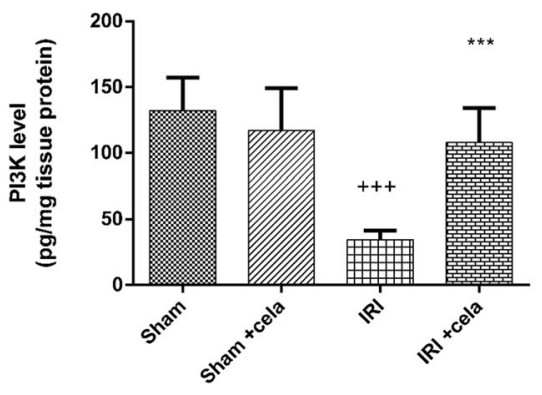

Fig. 3 Effect of celastrol treatment $(2 \mathrm{mg} / \mathrm{kg}$; PO) on the level of a $\mathrm{PI} 3 \mathrm{~K}$ and gene expression of $\mathbf{b} \mathrm{AKT}$ in renal IRI rats. All results were expressed as mean $\pm \mathrm{SD} ; n=6$ in each. PI3K phosphoinositide 3- b)

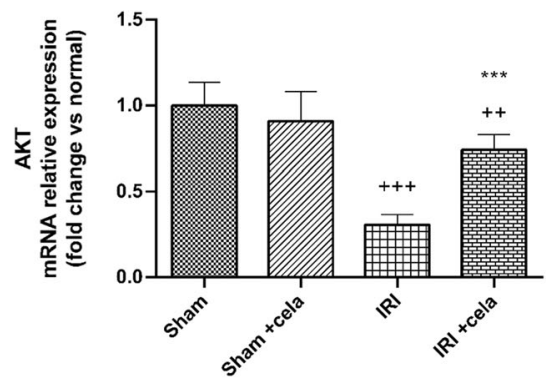

kinase, AKT protein kinase B, IRI ischemia-reperfusion injury. ${ }^{+++} P<0.001,{ }^{++} P<0.01$ compared with the sham group, ${ }^{* * *} P<$ 0.001 compared with the renal IRI group 
Fig. 4 Effect of celastrol treatment $(2 \mathrm{mg} / \mathrm{kg}$; $\mathrm{PO})$ on a P-ERK1/2:t-ERK1/2 ratio, levels of b NF-kBP65 and c I $\kappa B-\alpha$ in renal IRI rats. All results were expressed as mean $\pm \mathrm{SD} ; n=6$ each. ERK extracellular signal-regulated kinase, NF-kBP65 nuclear transcription factor-kappa B P65, IкB- $\alpha$ inhibitor of kappa B alpha, IRI ischemia-reperfusion injury. ${ }^{+++} P<0.001$ and ${ }^{++} P<$ 0.01 compared with the sham group, ${ }^{* * *} P<0.001$ and $* P<$ 0.05 compared with the renal IRI group

Fig. 5 Effect of celastrol treatment $(2 \mathrm{mg} / \mathrm{kg}$; PO) on levels of a IL-6, b TNF $\alpha$, and c cleaved caspase- 3 in renal IRI rats. All results were expressed as mean $\pm \mathrm{SD} ; n=6$ each. IL- 6 interleukin-6, TNF $\alpha$ tumor necrosis factor-alpha, IRI ischemia-reperfusion injury. ${ }^{++} P<0.001,{ }^{+} P<0.05$ compared with the sham group, $* * * P<0.001$ compared with the renal IRI group a)

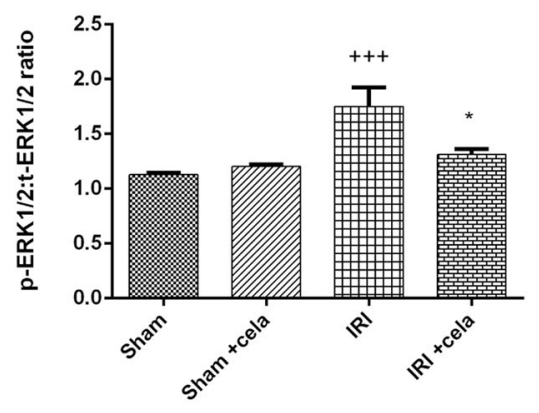

b)

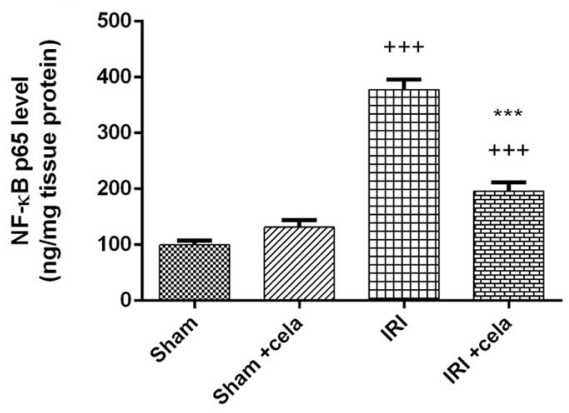

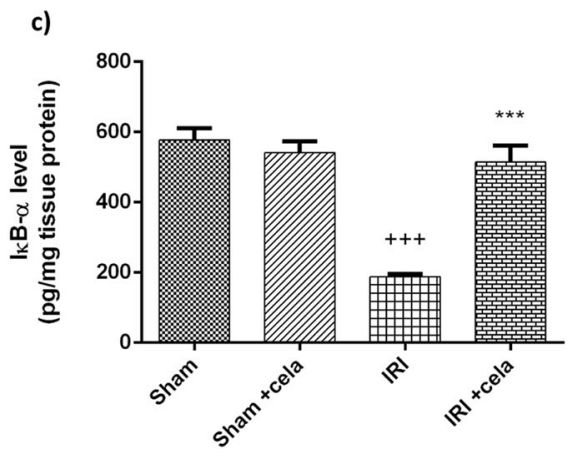

a)

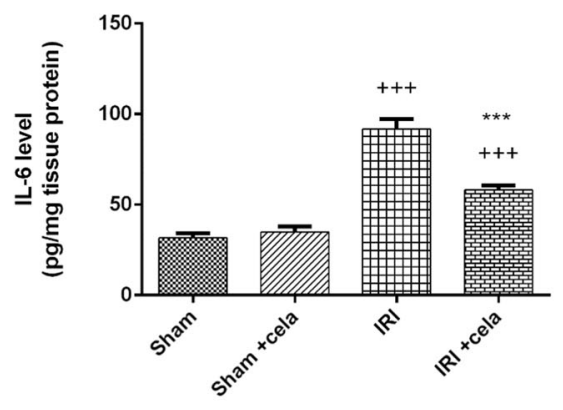

b)

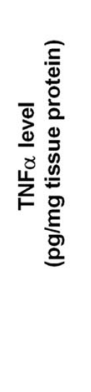

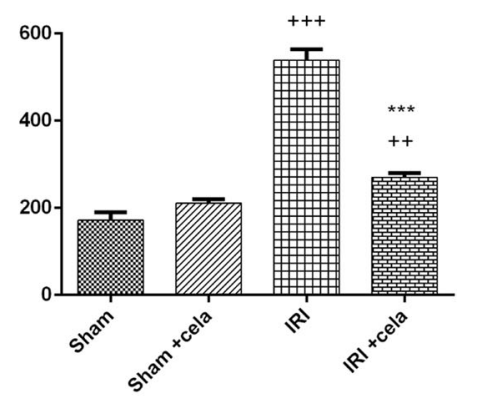

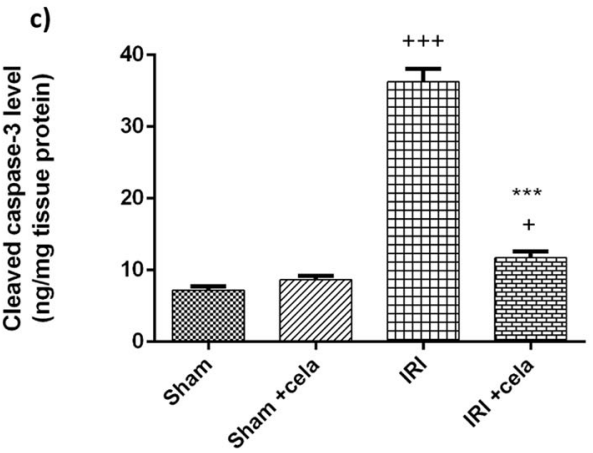

Celastrol Improved Histological Alterations Induced via Renal Ischemia-reperfusion

Kidney sections from sham-operated rats stained with $\mathrm{H}$ and E stain showed well-organized glomeruli (black arrows) and normal tubules (yellow arrow) (Fig. 6a). Additionally, sham-operated rats treated with cela showed mild disorganization of glomeruli and moderate congestion of its capillaries (black arrows), and the tubules are within normal (yellow arrows) (Fig. 6b). However, kidney sections from 

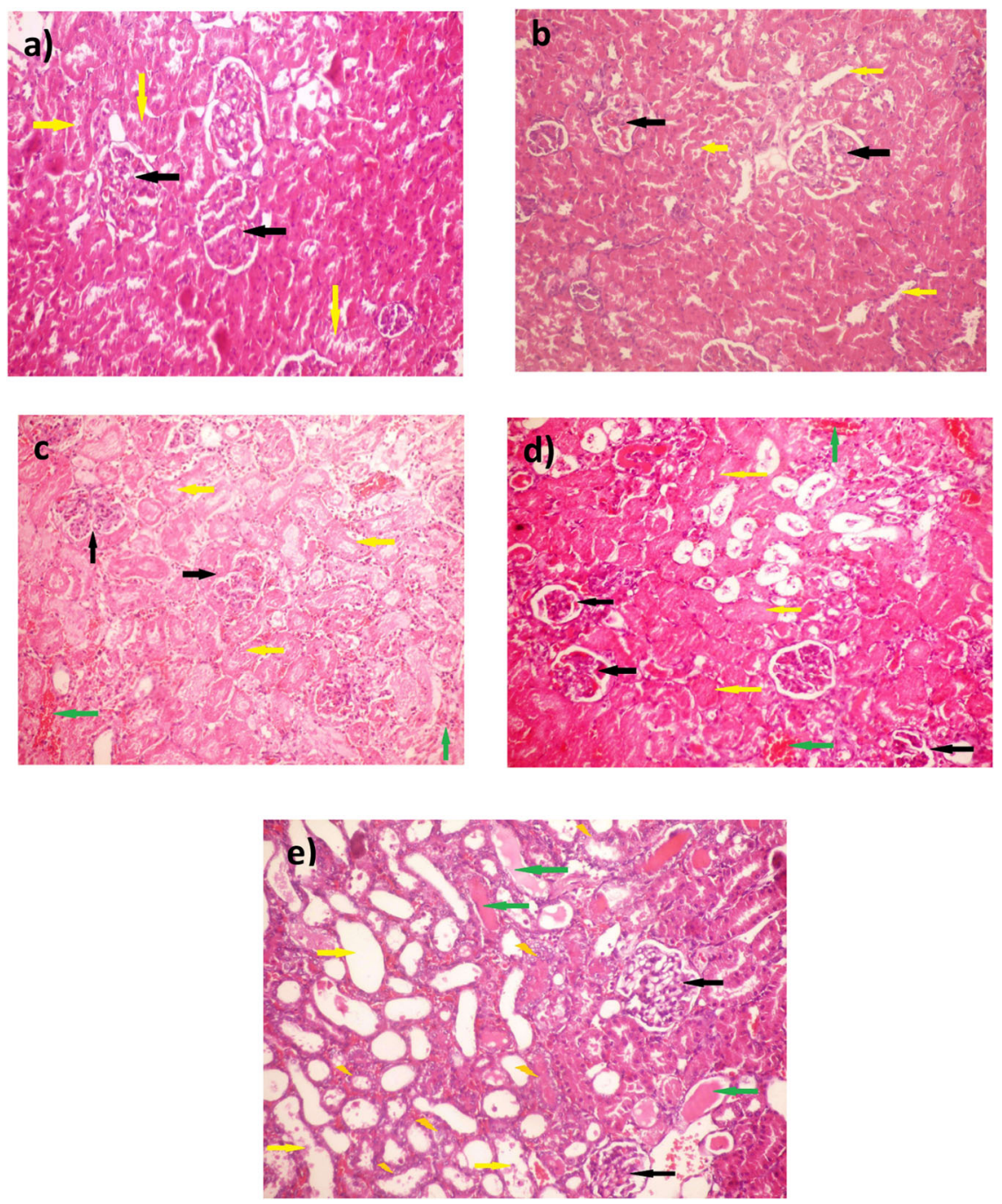

Fig. 6 Effect of celastrol treatment $(2 \mathrm{mg} / \mathrm{kg}$; PO) on H\&E-stained kidney sections of renal IRI rats. Representative microscopic pictures of H\&E-stained kidney sections showed well-organized glomeruli (black arrows) and normal tubules (yellow arrow) in sham-operated rats (a). Kidney sections from sham-operated rats treated with cela showed mild disorganization of the glomeruli with moderate congestion of its capillaries (black arrows), and the tubules are within normal (yellow arrows) (b). In kidney sections of renal IRI rats, most glomeruli were collapsed with focal necrosis (black arrows), the tubules

renal IRI rats displayed collapsed glomeruli with focal necrosis (black arrows), and the tubules showed marked necrosis with loss of the histologic details (yellow arrows). In addition, the stroma showed many foci of interstitial hemorrhage (green arrows) (Fig. 6c, d). Celastrol-treated rats' kidneys showed hyperplastic glomeruli with mild congestion (black arrows), some tubules showed sloughed epithelial cells (yellow arrows), and others showed hyaline casts (green arrows). Besides, regenerated tubules with active vesicular nuclei are detected between damaged tubules (crooked arrows) (Fig. 6e). showed marked necrosis with loss of the histologic details (yellow arrows), and the stroma showed many foci of interstitial hemorrhage (green arrows) (c, d). Kidney sections from renal IRI + cela treated rats showed Hyperplastic glomeruli with mild congestion (black arrows), some tubules showed sloughed epithelial cells (yellow arrows), others showed hyaline casts (green arrows), regenerated tubules with active vesicular nuclei are detected between damaged tubules (crooked arrows) (e) $(\mathrm{H} \& \mathrm{E} \times 200)$. H\&E hematoxylin and eosin, cela celastrol, IRI ischemia-reperfusion injury

Kidney sections from sham-operated rats stained with PAS stain showed well-organized glomeruli with good staining of the capillary's basement membrane with PAS stain (black arrow), and the tubules showed welldemarcated outer basement membrane and inner brush border (yellow arrows) (Fig. 7a). Also, kidney sections from sham-operated rats treated with cela showed PAS staining of the glomerular capillary basement membrane (black arrows) with a prominent PAS-positive brush border (yellow arrows) (Fig. 7b). Renal IRI rat's kidney showed wrinkling and thickening of capillary basement 

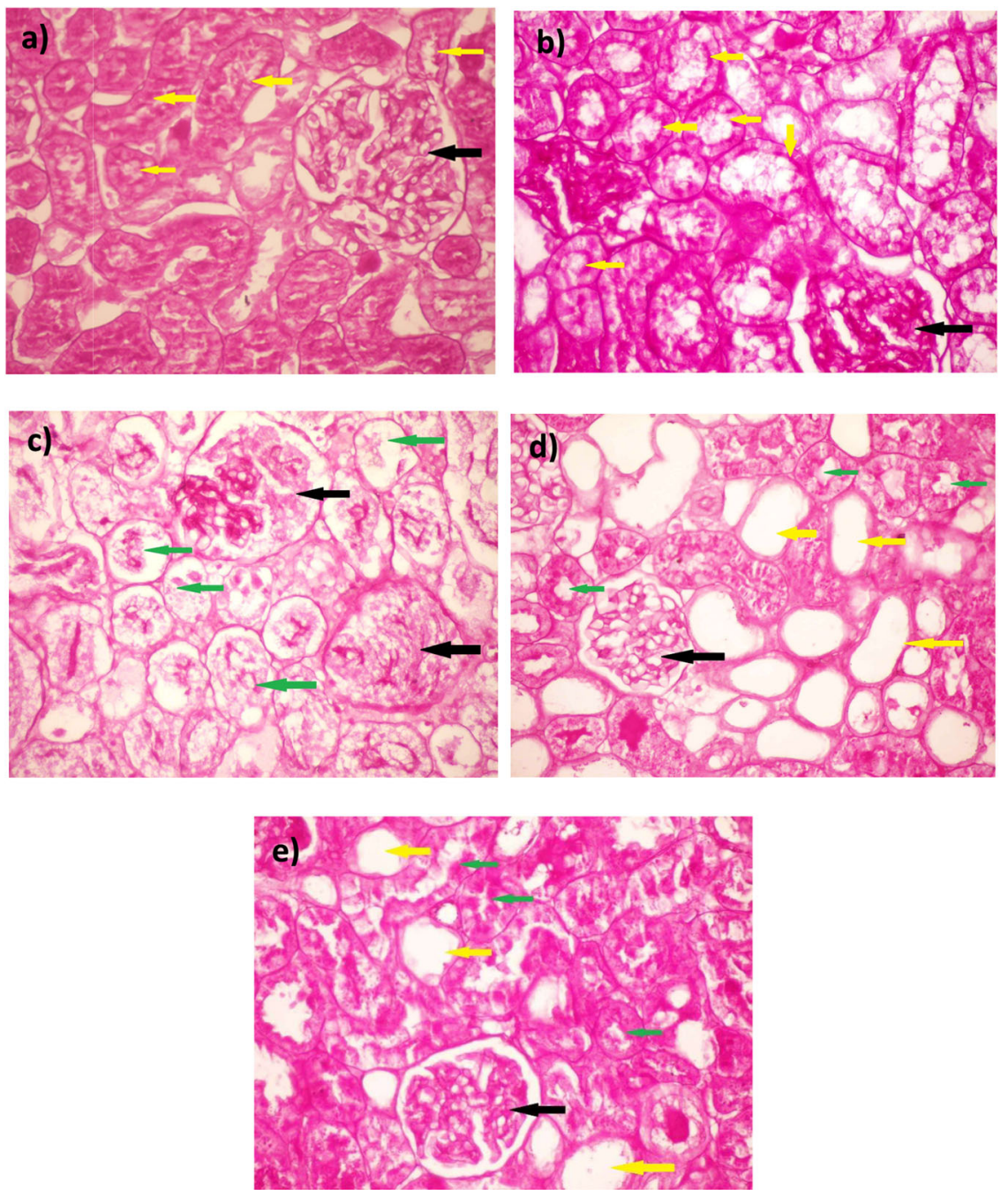

Fig. 7 Effect of celastrol treatment $(2 \mathrm{mg} / \mathrm{kg}$; PO) on PAS-stained kidney sections of renal IRI rats. Representative microscopic pictures of PAS-stained kidney sections showed well-organized glomeruli with good staining of the capillary's basement membrane with PAS stain (black arrow). In addition, the tubules showed well-demarcated outer basement membrane and inner brush border (yellow arrows) in shamoperated rats (a). Kidney sections from sham-operated rats treated with cela showed PAS staining of the glomerular capillaries' basement membrane (black arrows) with prominent PAS-positive brush border (yellow arrows) (b). kidney sections of renal IRI rats showed

membranes and collapse of the capillary lumen (black arrows) with significant tubular necrosis and cast formation (green arrows) (Fig. 7c). Kidney sections from IRI + cela treated rats showed hyperplastic glomeruli with the moderate demarcation of its capillaries' basement membrane (black arrow). Additionally, there were damaged tubules with loss of basement membrane and inner brush border (yellow arrows) and regenerated tubules with restoration of PAS staining of the basement membrane and brush border (green arrows) (Fig. 7d, e). wrinkling and thickening of capillary basement membranes and collapse of the capillary lumen (black arrows) with significant tubular necrosis and cast formation (green arrows) (c). Kidney sections from renal IRI + cela treated rats showed hyperplastic glomeruli with the moderate demarcation of its capillaries' basement membrane (black arrow). Damaged tubules lost their basement membrane and inner brush border (yellow arrows). Regenerated tubules with the restoration of PAS staining of the basement membrane and brush border (green arrows) (d, e) (PAS stain $\times 400$ ). PAS Periodic acid-Schiff, cela celastrol, IRI ischemia-reperfusion injury

\section{Discussion}

Celastrol has been studied for its protective effect against renal IRI in rats by inhibiting NF-kB activation [25]. However, in this study, we investigated the antioxidant potential of celastrol through its effect on Nrf2/HO-1 pathway. Furthermore, we explored celastrol's effect on PI3K/AKT cell survival pathway, ERK phosphorylation, NF-kB activation, and subsequent inflammatory and apoptotic responses. 
Our study revealed a significant decrease in MDA level and a significant increase in reduced GSH content in celastrol-treated rats compared with renal IRI rats. Renal IR is characterized by a massive ROS production that derives kidney cell damage and apoptosis [26]. Celastrol could preserve kidney cells from damage and correct the deteriorated kidney functions; serum creatinine and BUN in renal IR rats result from oxidative stress inhibition. The celastrol protective effect against renal injury was also proved by the significant downregulation of kidney injury molecule (KIM-1) gene expression compared with the IRI group. Histopathological observations confirmed these biochemical results. These results follow previous reports that proved celastrol's antioxidant activity in other kidney injury rat models [27, 28]. We tried underlining the possible mechanisms by which celastrol may exert its antioxidant potential by exploring the celastrol effect on Nrf2/ HO-1 pathway.

Nrf2 is a transcription factor that plays a protective role in experimental acute kidney injury through regulation of endogenous antioxidant enzyme gene expression and subsequent alleviation of oxidative stress [29]. Nrf2 -l- transgenic mice showed provoked kidney injury after IRI [30]. As well, Nrf2 is considered a key regulator of HO-1 gene expression [31]. HO-1 represents a protective system by targeting the pro-oxidant substrate; heme in a reaction generates the antioxidants biliverdin and bilirubin and the vasodilator molecule; carbon monoxide with antiapoptotic properties [32]. Our results showed an enhancement of Nrf2 gene expression and elevation in HO-1 levels in IRI rats as a defensive response to IR injury, and these results follow a previous study [33]. Meanwhile, celastrol-treated rats exhibited a significant upregulation of Nrf2 gene expression along with a significant increase in HO-1 renal level compared with renal IRI rats, indicating that celastrol exerts its protective role against renal IRI by activating Nrf2 with a subsequent increase in HO-1 renal level.

Our results suggested the protective effect of celastrol in renal IRI model via activation of the cell survival mediating pathway; PI3K/AKT indicated by a significant increase in PI3K renal level and remarkable upregulation of AKT gene expression in the ecelastrol-pretreated group compared with the IRI group. PI3K/AKT signaling pathway plays a significant role in protecting kidney cells against IRI [34, 35]. This protective effect has been attributed to its inhibitory effect on many inflammatory cytokines and pro-apoptotic mediators that evolved in response to kidney injury [36, 37]. Interestingly, it was reported that the PI3K/AKT pathway mediates the activation of Nrf2 nuclear translocation and subsequent HO-1 induction [38, 39]. Our results may provide further evidence that celastrol exerts its reno-protective effect by activating the Nrf2/HO-1 pathway through the PI3K/AKTdependent mechanism.

An ERK, one of the MAPK family members and NF- $\kappa \mathrm{B}$ transcription factors, is involved in renal IRI. Their activation is considered a key for inflammatory response and cell apoptosis regulation during the IR process [40, 41]. Furthermore, it has been reported that NF- $\mathrm{B}$ p65 subunit phosphorylation and acetylation are central in NF- $\kappa \mathrm{B}$ activation [42]. Therefore, we evaluated the phosphorylated, total ERK1/2, NF-kBP65, and the NF-kB inhibitor protein $(\mathrm{I} \kappa \mathrm{B}-\alpha)$ levels to estimate the role of celastrol in the modulation of MAPK and NF-kB signaling pathways and subsequent inflammatory cytokine (IL-6 and TNF $\alpha$ ) regulation. In addition, the key regulatory factor in apoptosis; cleaved caspase-3 level, was also determined. Remarkably, celastrol pretreatment displayed a notable inhibition of ERK 1/2 phosphorylation accompanied by a significant decrease in NF-kBP65 and an increase in I $\mathrm{BB}-\alpha$ levels with a subsequent decrease in IL-6, TNF $\alpha$, and cleaved caspase-3 levels compared with the renal IRI group.

Stimulation of MAPK signaling pathway comprising ERK1/2 phosphorylation activation is initiated by diverse inflammatory and stressful stimuli and is reported to be associated with inflammation and cell death during renal injury [43]. MAPK activation may also be closely related to $\mathrm{NF}-\kappa \mathrm{B}$ translocation to the nucleus and subsequent activation of various inflammatory and apoptotic cascades [44-46]. The master kinase in the toll-like receptor pathway and activated transforming growth factor betaactivated kinase 1 (TAK1) mediates IKK activation, which is essential in NF- $\kappa \mathrm{B}$ activation. Likewise, TAK1 mediates the activation of MAPKs [47]. This suggests interesting crosstalk between MAPK signaling and the NF$\kappa \mathrm{B}$ pathway. Along this line, studies have emphasized celastrol's inhibitory effect on MAPKs and NF-кB pathway, and thus suppression of inflammatory cytokines and pro-apoptotic mediators release in different rat models [48-51]. Consistently, our results showed that celastrol pretreatment possibly exerts its protective effect against IRI through inhibition of ERK phosphorylation and NF- $\kappa \mathrm{B}$ mediated inflammation and apoptosis.

\section{Conclusion}

Celastrol pretreatment showed a reno-protective potential against renal IRI by suppressing oxidative stress by enhancing the antioxidant defense system; Nrf2/HO-1. Also, celastrol showed activation of the survival signaling pathway; PI3K/AKT that protects renal tissue from IRI. In addition, Celastrol also displayed a significant antiinflammatory and antiapoptotic effect through the inhibition of NF- $\kappa \mathrm{B}$ activation and ERK phosphorylation, which 
suppressed their downstream effectors, inflammatory cytokines (IL-6 and TNF $\alpha$ ) and pro-apoptotic mediator (caspase-3). These findings prove celastrol is a promising candidate for protecting the kidney against IR injury with potential clinical application.

\section{Data availability}

The authors confirm that the data supporting the findings of this study are available upon request.

Acknowledgements The authors acknowledge the support given by Faculty of Pharmacy, Delta University for Science and Technology for using its animal unit services.

Author contributions All authors contribute in the project plan, laboratory work, data analysis and manuscript writing and revision before submission.

Funding This work was supported through the Annual Funding track by the Deanship of Scientific Research, Vice Presidency for Graduate Studies and Scientific Research, King Faisal University, Saudi Arabia [Project No. AN000018].

\section{Compliance with ethical standards}

Conflict of interest The authors declare no competing interests.

Ethical approval Rats care and experimental techniques were permitted by the Institutional Animal Care and Use Committee (IACUC) at Faculty of Pharmacy, Delta University for Science and Technology. Ethical approval number; FPDU 6/2019. All experiment protocols were conducted in harmony with the relevant guidelines and regulations.

Publisher's note Springer Nature remains neutral with regard to jurisdictional claims in published maps and institutional affiliations.

Open Access This article is licensed under a Creative Commons Attribution 4.0 International License, which permits use, sharing, adaptation, distribution and reproduction in any medium or format, as long as you give appropriate credit to the original author(s) and the source, provide a link to the Creative Commons license, and indicate if changes were made. The images or other third party material in this article are included in the article's Creative Commons license, unless indicated otherwise in a credit line to the material. If material is not included in the article's Creative Commons license and your intended use is not permitted by statutory regulation or exceeds the permitted use, you will need to obtain permission directly from the copyright holder. To view a copy of this license, visit http://creativecommons. org/licenses/by/4.0/.

\section{References}

1. Wang, Y., Li, C., Gu, J., Chen, C., Duanmu, J., \& Miao, J., et al. (2020). Celastrol exerts anti-inflammatory effect in liver fibrosis via activation of AMPK-SIRT3 signalling. Journal of Cellular and Molecular Medicine, 24(1), 941-953.
2. Gu, L., Bai, W., Li, S., Zhang, Y., Han, Y., \& Gu, Y., et al. (2013). Celastrol prevents atherosclerosis via inhibiting LOX-1 and oxidative stress. PloS One, 8(6), e65477.

3. Jannuzzi, A., Kara, M., \& Alpertunga, B. (2018). Celastrol ameliorates acetaminophen-induced oxidative stress and cytotoxicity in HepG2 cells. Human \& Experimental Toxicology, 37(7), 742-751.

4. Jung, H. W., Chung, Y. S., Kim, Y. S., \& Park, Y. K. (2007). Celastrol inhibits production of nitric oxide and proinflammatory cytokines through MAPK signal transduction and NF-kappaB in LPS-stimulated BV-2 microglial cells. Experimental \& Molecular Medicine, 39(6), 715-721.

5. Joshi, V., Venkatesha, S. H., Ramakrishnan, C., Nanjaraj Urs, A. N., Hiremath, V., \& Moudgil, K. D., et al. (2016). Celastrol modulates inflammation through inhibition of the catalytic activity of mediators of arachidonic acid pathway: Secretory phospholipase A2 group IIA, 5-lipoxygenase and cyclooxygenase-2. Pharmacological Research, 113(Pt A), 265-275.

6. Li, H., Zhang, Y. Y., Tan, H. W., Jia, Y. F., \& Li, D. (2008). Therapeutic effect of tripterine on adjuvant arthritis in rats. Journal of Ethnopharmacology, 118(3), 479-484.

7. Pinna, G. F., Fiorucci, M., Reimund, J. M., Taquet, N., Arondel, Y., \& Muller, C. D. (2004). Celastrol inhibits pro-inflammatory cytokine secretion in Crohn's disease biopsies. Biochemical and Biophysical Research Communications, 322(3), 778-786.

8. Shaker, M. E., Ashamallah, S. A., \& Houssen, M. E. (2014). Celastrol ameliorates murine colitis via modulating oxidative stress, inflammatory cytokines and intestinal homeostasis. Chemico-Biological Interactions, 210, 26-33.

9. Kim, D. Y., Park, J. W., Jeoung, D., \& Ro, J. Y. (2009). Celastrol suppresses allergen-induced airway inflammation in a mouse allergic asthma model. European Journal of Pharmacology, 612 (1-3), 98-105.

10. Liu, J., Lee, J., Salazar Hernandez, M. A., Mazitschek, R., \& Ozcan, U. (2015). Treatment of obesity with celastrol. Cell., 161 (5), 999-1011.

11. Chow, A. M., Tang, D. W., Hanif, A., \& Brown, I. R. (2014). Localization of heat shock proteins in cerebral cortical cultures following induction by celastrol. Cell Stress \& Chaperones, 19(6), 845-851.

12. Kim, J. E., Lee, M. H., Nam, D. H., Song, H. K., Kang, Y. S., \& Lee, J. E., et al. (2013). Celastrol, an NF-kappaB inhibitor, improves insulin resistance and attenuates renal injury in $\mathrm{db} / \mathrm{db}$ mice. PloS One, 8(4), e62068.

13. Sethi, G., Ahn, K. S., Pandey, M. K., \& Aggarwal, B. B. (2007). Celastrol, a novel triterpene, potentiates TNF-induced apoptosis and suppresses invasion of tumor cells by inhibiting NF-kappaBregulated gene products and TAK1-mediated NF-kappaB activation. Blood., 109(7), 2727-2735.

14. Saber, S., Ghanim, A. M., El-Ahwany, E., \& Abd El-Kader, E. M. (2020). Novel complementary antitumour effects of celastrol and metformin by targeting IкBкB, apoptosis and NLRP3 inflammasome activation in diethylnitrosamine-induced murine hepatocarcinogenesis. Cancer Chemotherapy and Pharmacology, 85(2), 331-343.

15. Shanmugam, M. K., Ahn, K. S., Lee, J. H., Kannaiyan, R., Mustafa, N., \& Manu, K. A., et al. (2018). Celastrol attenuates the invasion and migration and augments the anticancer effects of bortezomib in a xenograft mouse model of multiple myeloma. Frontiers in Pharmacology, 9, 365.

16. Tao, X., Younger, J., Fan, F. Z., Wang, B., \& Lipsky, P. E. (2002). Benefit of an extract of Tripterygium Wilfordii Hook F in patients with rheumatoid arthritis: a double-blind, placebocontrolled study. Arthritis and Rheumatism, 46(7), 1735-1743.

17. Kitzen, J. J., de Jonge, M. J., Lamers, C. H., Eskens, F. A., van der Biessen, D., \& van Doorn, L., et al. (2009). Phase I doseescalation study of F60008, a novel apoptosis inducer, in patients 
with advanced solid tumours. European Journal of Cancer, 45 (10), 1764-1772.

18. Ge, Y., Xie, H., Li, S., Jin, B., Hou, J., \& Zhang, H., et al. (2013). Treatment of diabetic nephropathy with Tripterygium wilfordii Hook F extract: a prospective, randomized, controlled clinical trial. Journal of Translational Medicine, 11, 134.

19. Ji, S. M., Wang, Q. W., Chen, J. S., Sha, G. Z., Liu, Z. H., \& Li, L. S. (2006). Clinical trial of Tripterygium Wilfordii Hook F. in human kidney transplantation in China. Transplantation Proceedings, 38(5), 1274-1279.

20. Zhang, M., Chen, Y., Yang, M. J., Fan, X. R., Xie, H., \& Zhang, L., et al. (2019). Celastrol attenuates renal injury in diabetic rats via MAPK/NF-kappaB pathway. Phytotherapy Research, 33(4), 1191-1198.

21. Tang, M., Cao, X., Zhang, K., Li, Y., Zheng, Q. Y., \& Li, G. Q., et al. (2018). Celastrol alleviates renal fibrosis by upregulating cannabinoid receptor 2 expression. Cell Death \& Disease, 9(6), 601.

22. Yu, X., Meng, X., Xu, M., Zhang, X., Zhang, Y., \& Ding, G., et al. (2018). Celastrol ameliorates cisplatin nephrotoxicity by inhibiting NF-kappaB and improving mitochondrial function. EBioMedicine., 36, 266-280.

23. Chu, C., He, W., Kuang, Y., Ren, K., \& Gou, X. (2014). Celastrol protects kidney against ischemia-reperfusion-induced injury in rats. The Journal of Surgical Research, 186(1), 398-407.

24. Rusai, K., Sollinger, D., Baumann, M., Wagner, B., Strobl, M., \& Schmaderer, C., et al. (2010). Toll-like receptors 2 and 4 in renal ischemia/reperfusion injury. Pediatric Nephrology, 25(5), 853-860.

25. Chu, C., He, W., Kuang, Y., Ren, K., \& Gou, X. (2014). Celastrol protects kidney against ischemia-reperfusion-induced injury in rats. Journal of Surgical Research, 186(1), 398-407.

26. Malek, M., \& Nematbakhsh, M. (2015). Renal ischemia/reperfusion injury; from pathophysiology to treatment. J Renal Inj Prev, 4(2), 20-27.

27. Kim J. E., Lee M. H., Nam D. H., Song H. K., Kang Y. S., Lee J. E., et al. Celastrol, an NF- $\mathrm{kB}$ inhibitor, improves insulin resistance and attenuates renal injury in $\mathrm{db} / \mathrm{db}$ mice. PloS One. 2013 Apr 26;8(4):e62068

28. Wu, M., Chen, W., Yu, X., Ding, D., Zhang, W., \& Hua, H., et al. (2018). Celastrol aggravates LPS-induced inflammation and injuries of liver and kidney in mice. American Journal of Translational Research, 10(7), 2078.

29. Copple, I. M., Lister, A., Obeng, A. D., Kitteringham, N. R., Jenkins, R. E., \& Layfield, R., et al. (2010). Physical and functional interaction of sequestosome 1 with Keap1 regulates the Keap1-Nrf2 cell defense pathway. Journal of Biological Chemistry, 285(22), 16782-16788.

30. Liu, M., Grigoryev, D. N., Crow, M. T., Haas, M., Yamamoto, M., \& Reddy, S. P., et al. (2009). Transcription factor Nrf2 is protective during ischemic and nephrotoxic acute kidney injury in mice. Kidney International, 76(3), 277-285.

31. Zhang, H., Liu, Y.-Y., Jiang, Q., Li, K.-R., Zhao, Y.-X., \& Cao, C., et al. (2014). Salvianolic acid A protects RPE cells against oxidative stress through activation of Nrf2/HO-1 signaling. Free Radical Biology and Medicine, 69, 219-228.

32. Shah, Z. A., Li, R.-C., Ahmad, A. S., Kensler, T. W., Yamamoto, M., \& Biswal, S., et al. (2010). The Flavanol (-)-Epicatechin Prevents Stroke Damage through the Nrf2/HO1 Pathway. Journal of Cerebral Blood Flow \& Metabolism, 30(12), 1951-1961.

33. Shelton, L. M., Kevin Park, B., \& Copple, I. M. (2013). Role of Nrf2 in protection against acute kidney injury. Kidney International, 84(6), 1090-1095.

34. Zhang, G., Wang, Q., Zhou, Q., Wang, R., Xu, M., \& Wang, H., et al. (2016). Protective effect of tempol on acute kidney injury through PI3K/Akt/Nrf2 signaling pathway. Kidney and Blood Pressure Research, 41(2), 129-138.

35. Wei, Q., Zhao, J., Zhou, X., Yu, L., Liu, Z., \& Chang, Y. (2019). Propofol can suppress renal ischemia-reperfusion injury through the activation of PI3K/AKT/mTOR signal pathway. Gene., 708, 14-20.

36. Yu, J.-B., Shi, J., Zhang, Y., Gong, L.-R., Dong, S.-A., \& Cao, X.-S., et al. (2015). Electroacupuncture Ameliorates Acute Renal Injury in Lipopolysaccharide-Stimulated Rabbits via Induction of HO-1 through the PI3K/Akt/Nrf2 Pathways. PloS One, 10(11), e0141622-e.

37. Mohamed, A. F., Safar, M. M., Zaki, H. F., \& Sayed, H. M. (2017). Telluric Acid Ameliorates Endotoxemic Kidney Injury in Mice: Involvement of TLR4, Nrf2, and PI3K/Akt Signaling Pathways. Inflammation., 40(5), 1742-1752.

38. Hamdulay, S. S., Wang, B., Birdsey, G. M., Ali, F., Dumont, O., \& Evans, P. C., et al. (2010). Celecoxib activates PI-3K/Akt and mitochondrial redox signaling to enhance heme oxygenase-1mediated anti-inflammatory activity in vascular endothelium. Free Radical Biology and Medicine, 48(8), 1013-1023.

39. Ali, T., Kim, T., Rehman, S. U., Khan, M. S., Amin, F. U., \& Khan, M., et al. (2018). Natural Dietary Supplementation of Anthocyanins via PI3K/Akt/Nrf2/HO-1 Pathways Mitigate Oxidative Stress, Neurodegeneration, and Memory Impairment in a Mouse Model of Alzheimer's Disease. Molecular Neurobiology, 55(7), 6076-6093.

40. Xu, Z. J., Shu, S., Li, Z. J., Liu, Y. M., Zhang, R. Y., \& Zhang, Y. (2017). Liuwei Dihuang pill treats diabetic nephropathy in rats by inhibiting of TGF- $\beta$ /SMADS, MAPK, and NF-kB and upregulating expression of cytoglobin in renal tissues. Medicine, 96(3), e5879-e5879.

41. Alderliesten, M., de Graauw, M., Oldenampsen, J., Qin, Y., Pont, C., \& van Buren, L., et al. (2007). Extracellular Signal-Regulated Kinase Activation during Renal Ischemia/Reperfusion Mediates Focal Adhesion Dissolution and Renal Injury. The American Journal of Pathology, 171(2), 452-462.

42. Islam, K. N., Bae, J.-W., Gao, E., \& Koch, W. J. (2013). Regulation of nuclear factor $\kappa \mathrm{B}(\mathrm{NF}-\mathrm{\kappa B})$ in the nucleus of cardiomyocytes by $\mathrm{G}$ protein-coupled receptor kinase 5 (GRK5). Journal of Biological Chemistry, 288(50), 35683-35689.

43. Kumar, S., Allen, D. A., Kieswich, J. E., Patel, N. S., Harwood, S., \& Mazzon, E., et al. (2009). Dexamethasone ameliorates renal ischemia-reperfusion injury. Journal of the American Society of Nephrology, 20(11), 2412-2425.

44. Zhang, J., Xia, J., Zhang, Y., Xiao, F., Wang, J., \& Gao, H., et al. (2016). HMGB1-TLR4 signaling participates in renal ischemia reperfusion injury and could be attenuated by dexamethasonemediated inhibition of the ERK/NF- $\mathrm{BB}$ pathway. American Journal of Translational Research, 8(10), 4054-4067.

45. Ramalingam, P., Poulos, M. G., Lazzari, E., Gutkin, M. C., Lopez, D., \& Kloss, C. C., et al. (2020). Chronic activation of endothelial MAPK disrupts hematopoiesis via NFKB dependent inflammatory stress reversible by SCGF. Nature. Communications., 11(1), 666.

46. Li, Z., Nickkholgh, A., Yi, X., Bruns, H., Gross, M.-L., \& Hoffmann, K., et al. (2009). Melatonin protects kidney grafts from ischemia/reperfusion injury through inhibition of NF-kB and apoptosis after experimental kidney transplantation. Journal of Pineal Research, 46(4), 365-372.

47. Shi J.-H., Sun S.-C. Tumor necrosis factor receptor-associated factor regulation of nuclear factor $\kappa \mathrm{b}$ and mitogen-activated protein kinase Pathways. Frontiers in Immunology. 2018 Aug 9;9:1849

48. Luo, D., Guo, Y., Cheng, Y., Zhao, J., Wang, Y., \& Rong, J. (2017). Natural product celastrol suppressed macrophage M1 polarization against inflammation in diet-induced obese mice via regulating Nrf2/HO-1, MAP kinase and NF-кB pathways. Aging, 9(10), 2069-2082. 
49. Dai, W., Wang, X., Teng, H., Li, C., Wang, B., \& Wang, J. (2019). Celastrol inhibits microglial pyroptosis and attenuates inflammatory reaction in acute spinal cord injury rats. International Immunopharmacology, 66, 215-223.

50. Zhang, M., Chen, Y., Yang, M.-J., Fan, X.-R., Xie, H., \& Zhang, L., et al. (2019). Celastrol attenuates renal injury in diabetic rats via MAPK/NF-кB pathway. Phytotherapy Research, 33(4), 1191-1198.

51. Yu, X., Meng, X., Xu, M., Zhang, X., Zhang, Y., \& Ding, G., et al. (2018). Celastrol ameliorates cisplatin nephrotoxicity by inhibiting NF- $\mathrm{KB}$ and improving mitochondrial function. EBioMedicine., 36, 266-280. 